\title{
Pathophysiological Commonality Between Irritable Bowel Syndrome and Metabolic Syndrome: Role of Corticotropin-releasing Factor-Toll-like Receptor 4-Proinflammatory Cytokine Signaling
}

\author{
Tsukasa Nozu ${ }^{1,2 *}$ and Toshikatsu Okumura, \\ ${ }^{I}$ Department of Regional Medicine and Education, Asahikawa Medical University, Asahikawa, Hokkaido, Japan; ${ }^{2}$ Center for Medical Education, \\ Asahikawa Medical University, Asahikawa, Hokkaido, Japan; ${ }^{3}$ Division of Gastroenterology and Hematology/Oncology, Department of Medicine, \\ Asahikawa Medical University, Asahikawa, Hokkaido, Japan; and ${ }^{4}$ Department of General Medicine, Asahikawa Medical University, Asahikawa, \\ Hokkaido, Japan
}

Irritable bowel syndrome (IBS) displays chronic abdominal pain with altered defecation. Most of the patients develop visceral hypersensitivity possibly resulting from impaired gut barrier and altered gut microbiota. We previously demonstrated that colonic hyperpermeability with visceral hypersensitivity in animal IBS models, which is mediated via corticotropin-releasing factor (CRF)-Tolllike receptor 4 (TLR4)-proinflammatory cytokine signaling. CRF impairs gut barrier via TLR4. Leaky gut induces bacterial translocation resulting in dysbiosis, and increases lipopolysaccharide (LPS). Activation of TLR4 by LPS increases the production of proinflammatory cytokines, which activate visceral sensory neurons to induce visceral hypersensitivity. LPS also activates CRF receptors to further increase gut permeability. Metabolic syndrome (MS) is a cluster of cardiovascular risk factors, including insulin resistance, obesity, dyslipidemia, and hypertension, and recently several researchers suggested the possibility that impaired gut barrier and dysbiosis with low-grade systemic inflammation are involved in MS. Moreover, TLR4-proinflammatory cytokine contributes to the development of insulin resistance and obesity. Thus, the existence of pathophysiological commonality between IBS and MS is expected. This review discusses the potential mechanisms of IBS and MS with reference to gut barrier and microbiota, and explores the possibility of existence of a pathophysiological link between these diseases with a focus on CRF, TLR4, and proinflammatory cytokine signaling. We also review epidemiological data supporting this possibility, and discuss the potential of therapeutic application of the drugs used for MS to IBS treatment. This notion may pave the way for exploring novel therapeutic approaches for these disorders.

(J Neurogastroenterol Motil 2022;28:173-184)

Key Words

Gut barrier; Irritable bowel syndrome; Metabolic syndrome; Microbiota; Toll-like receptor 4

Received: January 4, 2021 Revised: August 26, 2021 Accepted: October 11, 2021

(a) This is an Open Access article distributed under the terms of the Creative Commons Attribution Non-Commercial License (http://creativecommons. org/licenses/by-nc/4.0) which permits unrestricted non-commercial use, distribution, and reproduction in any medium, provided the original work is properly cited.

${ }^{*}$ Correspondence: Tsukasa Nozu, MD, PhD, FACP, FJSIM

Department of Regional Medicine and Education, Asahikawa Medical University, 2-1-1-1 Midorigaoka-Higashi, Asahikawa, Hokkaido 078-8510, Japan

Tel: +81-166-68-2844, Fax: +81-166-68-2846, E-mail: tnozu@sea.plala.or.jp 


\section{Introduction}

Irritable bowel syndrome (IBS) displays chronic abdominal pain with altered defecation, which is not explained by structural or biochemical abnormalities, possibly resulting from impaired gastrointestinal (GI) function, such as disturbance of GI motility and visceral hypersensitivity. ${ }^{1}$ The prevalence of IBS in general population is between $10 \%$ to $20 \%$, and it impairs patients' quality of life and has a huge economic impact including direct costs of health care use and indirect costs of absenteeism from work. ${ }^{1}$ IBS is a stressrelated functional GI disorder, and stress significantly impacts on the development of IBS symptoms by altering colonic sensorimotor function. ${ }^{2,3}$ Corticotropin-releasing factor (CRF), which is a main molecule mediating stress-induced enhanced colonic motility and visceral hypersensitivity, is thought to be a vital contributor to the pathophysiology of IBS. ${ }^{2-4}$

Metabolic syndrome (MS) is a cluster of cardiovascular risk factors, including insulin resistance, obesity, dyslipidemia and hypertension, and it remains a major cause of mortality and morbidity. ${ }^{5}$ Recently, it has been shown that gut significantly contributes to the pathophysiology of MS, ie, altered gut microbiota and leaky gut with low-grade systemic inflammation, ${ }^{6}$ and chronic inflammationinduced insulin resistance is thought to be a crucial mechanism of MS. ${ }^{7}$ Additionally, lipopolysaccharide (LPS)-induced metabolic endotoxemia, and activation of Toll-like receptor 4 (TLR4) followed by increased production of proinflammatory cytokines are known to contribute to the development of insulin resistance and obesity. ${ }^{6,8}$

At the same time, it has been generally accepted the importance of altered microbiota and impaired gut integrity in the IBS pathophysiology. ${ }^{9,10}$ Moreover, we have shown that leaky gut manifested by colonic hyperpermeability observed in animal IBS models is mediated via TLR4 and proinflammatory cytokine signaling. ${ }^{11}$ Interestingly, CRF modulates TLR4-proinflammatory cytokine signaling to induce colonic hyperpermeability and visceral hypersensitivity. ${ }^{11}$

Thus, these lines of evidence may raise the notion that IBS and MS may have common mechanisms, which are mediated via CRF, TLR4, and proinflammatory cytokine signaling. In this paper, we review the mechanisms of IBS and MS with reference to gut barrier and microbiota, and discuss the commonality of pathophysiological mechanisms of these diseases with a focus on CRF, TLR4, and proinflammatory cytokine. Finally, the potential of therapeutic application of the drugs used for MS to IBS treatment is also discussed.

\section{Brain-gut Interaction in Irritable Bowel Syndrome}

Although the definite mechanisms of IBS have not been determined so far, altered GI function along with the disturbance of bidirectional communication between brain and gut, ie, braingut axis, is thought to be an important contributor to the symptom generation of IBS. ${ }^{3,12,13}$ Signal from the brain to gut modulates GI function, and IBS patients have higher reactivity to stress. ${ }^{14}$ Stress induces GI functional changes via CRF signaling, possibly leading to the development and exacerbation of IBS symptoms. ${ }^{2} \mathrm{CRF}$ is one of the important signaling molecules between brain and gut, and IBS patients have an exaggerated response to CRF on adrenocorticotropic hormone release and intestinal motility changes. ${ }^{15}$ Conversely, signal from the gut to brain modulates behavior and brain function. ${ }^{16}$ Actually, psychiatric comorbidities such as depression and anxiety are frequently observed in IBS patients. ${ }^{17}$

Abnormal GI motility and visceral hypersensitivity are known to be major GI functional changes in IBS, as described before. Several studies indicated that abnormal motility patterns are detected in IBS, but they are not constant, and no specific abnormality has been shown. Furthermore, only few studies have shown a link between abnormal motility and abdominal pain. ${ }^{18,19}$

On the other hand, most of the IBS patients display visceral hypersensitivity, ${ }^{20,21}$ and visceral pain threshold is correlated with IBS symptom severity. ${ }^{22}$ Additionally, we previously showed that stressinduced visceral hypersensitivity is a reliable marker for IBS. ${ }^{23}$ Thus, visceral hypersensitivity has been now generally accepted to be one of the most important pathophysiological features of IBS. ${ }^{19,24}$

\section{Impaired Gut Barrier and Activated Immune System in Irritable Bowel Syndrome}

In addition to these GI sensorimotor abnormalities, the importance of an impaired gut barrier associated with abnormal immune response in the pathophysiology of IBS has been recently recognized. ${ }^{2}$ Abnormal gut integrity manifested by increased gut permeability has been found in IBS. ${ }^{10,25}$ Gut hyperpermeability induces bacterial translocation, followed by activation of immune system leading to inflammation. In this process, LPS is released and triggers to produce proinflammatory cytokines via TLR $4 .{ }^{26}$ Actually, the increased levels of plasma proinflammatory cytokines and serum LPS are observed in IBS. ${ }^{26-28}$ Moreover, LPS-induced stimulation of proinflammatory cytokine release from peripheral 
blood mononuclear cells is enhanced in IBS, and higher symptoms severity such as urgency, diarrhea, etc, is associated with higher cytokine response induced by LPS. ${ }^{29}$ We previously demonstrated that LPS, interleukin (IL)-1 $\beta$, and IL-6 induce visceral hypersensitivity, ${ }^{30}$ suggesting that these inflammatory processes cause visceral pain in IBS.

At the same time, increased infiltration of mast cells in the gut mucosa is observed in IBS, and mast cell mediators, such as histamine and tryptase, which can increase gut permeability, are also increased in the colon of IBS. ${ }^{10}$ Additionally, mast cells have TLR4, and its activation increases the production of proinflammatory cytokines. ${ }^{31}$ Moreover, immunocytes such as macrophage and $\mathrm{T}$ cells are also reported to be increased in IBS. ${ }^{32}$

Although visceral hypersensitivity is thought to result from multifactorial processes, ${ }^{24}$ impaired gut barrier may be one of the causes. Knockdown of occludin, which is one of the tight junction proteins (TJPs) maintaining gut epithelial barrier, induces intestinal hyperpermeability with visceral hypersensitivity in mice. ${ }^{33}$ Moreover, we have recently confirmed that visceral hypersensitivity occurs associated with colonic hyperpermeability without exception in several IBS models, ${ }^{11,34-39}$ which may further support the notion above. Therefore, impaired gut barrier with enhanced LPS (TLR4)-proinflammatory cytokine signaling causing visceral hypersensitivity is thought to be a vital mechanism of IBS.

\section{Corticotropin-releasing Factor and Irritable Bowel Syndrome}

$\mathrm{CRF}$ is a principal initiator of stress response, which is released from the hypothalamus. CRF stimulates the pituitary-adrenal axis, and modulates behavior and autonomic nervous system activity to regulate GI function. ${ }^{2}$ Additionally, the expression of CRF receptors and ligands are detected in peripheral organs including GI tract, and peripheral CRF signaling is also activated under stress conditions. $^{3}$

Both brain and peripheral CRF modify GI motility, and stress-induced altered GI motility is well known to be mediated via $\mathrm{CRF}^{2,3}$ Fukudo et al, ${ }^{15}$ showed that peripheral administration of $\mathrm{CRF}$ also impairs intestinal motility in humans, and this response is exaggerated in IBS patients.

Furthermore, CRF induces visceral hypersensitivity in animals and human, ${ }^{2,3,40}$ and stress, such as restraint or water avoidance stress (WAS) causes visceral hypersensitivity, which is inhibited by $\mathrm{CRF}$ antagonists. ${ }^{3} \mathrm{CRF}$ also increases gut permeability, ${ }^{2}$ and neonatal maternal deprivation, restraint stress, or WAS-induced colonic hyperpermeability is mediated via CRF. ${ }^{11,41-44}$

$\mathrm{CRF}$ and its related peptides, urocortins (Ucns) exert their action through the activation of 2 receptors, $\mathrm{CRF}$ receptor type 1 $\left(\mathrm{CRF}_{1}\right)$ and type $2\left(\mathrm{CRF}_{2}\right){ }^{45,46}$ Each $\mathrm{CRF}$ receptor has a distinct role. Stress-induced enhanced colonic motility and visceral hypersensitivity are $\mathrm{CRF}_{1}$-dependent, and $\mathrm{CRF}_{2}$ inhibits these $\mathrm{CRF}_{1}$ triggered responses, and the activity balance of each subtype signaling determines the colonic functions in response to stress, which is designated as balance theory of CRF signaling. ${ }^{3,47}$

Interestingly, we have recently demonstrated that both increased colonic permeability and visceral hypersensitivity induced by CRF are mediated via TLR4 and IL-1. ${ }^{11}$ Moreover, LPS or repeated WAS induces colonic hyperpermeability and visceral hypersensitivity via CRF, TLR4, IL-1, and IL-6. ${ }^{11,30,48}$ Furthermore, activation of TLR4-proinflammatory cytokine signaling by CRF is $\mathrm{CRF}_{1}$-dependent, and stimulation of $\mathrm{CRF}_{2}$ blocks it, which follows the balance theory of CRF signaling. ${ }^{11,30}$ Therefore, CRFTLR4-proinflammatory cytokine signaling is considered to be a key pathway to impair gut barrier to induce visceral hypersensitivity, and the CRF signaling balance may be abnormally shifted to $\mathrm{CRF}_{1}$ in IBS ${ }^{3}$

Incidentally, mast cells have CRF receptors, and CRF triggers to release chemical mediators, such as serotonin, cytokines, etc, ${ }^{49}$ which may also contribute to impaired gut barrier and visceral hypersensitivity. ${ }^{10,50}$

\section{Altered Gut Microbiota in Irritable Bowel Syndrome}

Ample evidence suggests that altered microbiota plays a pivotal role in the IBS pathophysiology. Several studies have shown the alteration in gut microbiota in IBS. ${ }^{51}$ Moreover, the severity of symptoms of IBS is negatively associated with microbial richness in the gut. ${ }^{52}$ In animal studies, chronic stress models such as maternal separation and repeated WAS, which are also known to be IBS models displaying visceral hypersensitivity, can alter gut microbiota composition. ${ }^{53,54}$ Additionally, this microbial imbalance, ie, dysbiosis is mediated via increasing circulating LPS and proinflammatory cytokines levels, ${ }^{55,56}$ disrupting the intestinal barrier, ${ }^{57}$ and increasing the activity of the hypothalamus-pituitary-adrenal (HPA) axis triggered by $\mathrm{CRF}^{58}$ These lines of evidence suggest that impaired gut barrier via activated CRF-TLR4-proinflammatory cytokine system may alter microbiota, and consequently causes visceral hypersensitivity.

On the other hand, microbiota and their metabolites are one of 
the main contributors to the integrity of gut epithelial barrier, and dysbiosis can disrupt the epithelial barrier to increase gut permeability. ${ }^{59}$ Butyrate, which is one of the short-chain fatty acids (SCFAs) derived from gut bacteria, increases the expression of TJPs and helps in maintenance of gut barrier function. ${ }^{60}$ We previously demonstrated that intracolonic instillation of butyrate can inhibit repeated WAS-induced visceral hypersensitivity and colonic hyperpermeability in rats. ${ }^{38}$ Moreover, butyrate also blocks these GI

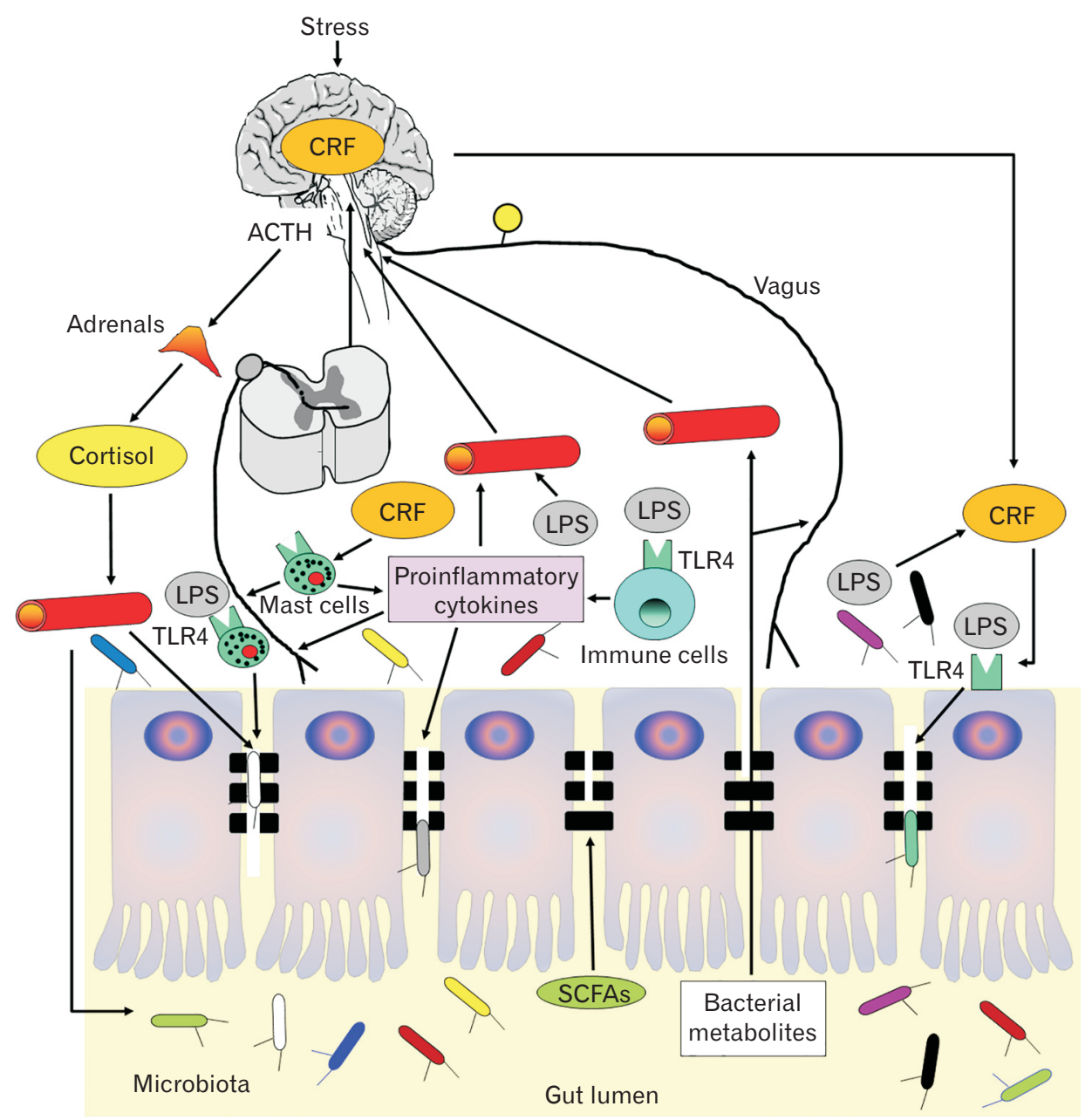

Figure 1. Schematic illustration of the candidate mechanisms of irritable bowel syndrome (IBS) with a focus on corticotropin-releasing factor (CRF), Toll-like receptor 4 (TLR4), and proinflammatory cytokine signaling. Hypothalamus - pituitary-adrenal (HPA) axis is activated by stress, which is triggered by CRF. Cortisol is produced from the adrenals to alter microbiota, and impairs gut barrier via modifying tight junction protein (TJP). Bacterial metabolites including short-chain fatty acids (SCFAs) also modify gut barrier integrity, and modulate brain and behavior via the vagus and/or directly acting brain through circulation, thereby possibly causing psychiatric comorbidities, which frequently occurs in IBS. $\mathrm{CRF}$ is also released from peripheral tissue including gastrointestinal (GI) tract triggered by stress, and acts peripheral CRF receptors. Peripheral $\mathrm{CRF}$ secretion is controlled by the brain possibly via the autonomic nerve. Activation of peripheral CRF receptors modifies TJP via TLR4 to increase colonic permeability. Then, bacterial translocation occurs leading to dysbiosis, and increases lipopolysaccharide (LPS). In turn, LPS activates TLR4 in immune cells to trigger the production of proinflammatory cytokines, which induce visceral hypersensitivity through the activation of visceral afferents, and impair gut barrier via modifying TJP. At the same time, mast cells having CRF receptors and TLR4, release chemical mediators including proinflammatory cytokines triggered by CRF and LPS, which can also induce these GI changes. LPS and proinflammatory cytokines can enter the circulation to act brain to alter emotion and cognition, which possibly contribute to psychiatric comorbidities in IBS. Moreover, proinflammatory cytokine also stimulates the secretion of CRF from hypothalamus leading to the activation of HPA axis. Additionally, LPS activates peripheral CRF receptors to further increase colonic permeability. Thus, CRF and TLR4-proinflammatory cytokine signaling create a vicious cycle resulting in leaky gut and dysbiosis to cause the symptoms of IBS. ACTH, adrenocorticotropic hormone. 
changes induced by CRF or LPS. ${ }^{38}$ At the same time, Zhang et $\mathrm{al}{ }^{61}$ reported that repeated WAS decreases occludin expression with reduced butyrate-producing microbiota, and this change of TJP is reversed by the supplementation of butyrate-producing bacteria. Additionally, the microbiota from IBS patients induces visceral hypersensitivity in germ-free rats, while the microbiota from healthy individuals does not. $^{62}$

Thus, microbiota can alter gut barrier via their metabolites, which seems to be one of the mechanisms that dysbiosis impairs gut barrier. In other words, altered microbiota and impaired gut barrier are thought to be a cause and consequent, which are mediated via CRF-TLR4-proinflammatory cytokine signaling.

\section{The Candidate Mechanisms of Irritable Bow- el Syndrome With a Focus on Corticotropin- releasing Factor, Toll-like Receptor 4, and Proinflammatory Cytokine Signaling}

As described before, repeated WAS induces visceral hypersensitivity and colonic hyperpermeability in rats. ${ }^{11,30}$ Moreover, we also demonstrated that peripheral injection of LPS or CRF mimics these GI changes, which simulates IBS pathophysiology. These GI changes in the IBS models above are mediated by CRF, TLR4, IL-1, and IL-6. ${ }^{11,30}$ According to these results, together with the evidence demonstrated so far, we speculate the candidate mechanisms of IBS as follows (Fig. 1).

Stress activates central CRF signaling to stimulate HPA axis. Cortisol is released from adrenals to alter microbiota. ${ }^{58}$ Additionally, cortisol also impairs gut barrier via modifying $\mathrm{TJP}^{63} \mathrm{SCFAs}^{\mathrm{S}}$ produced by bacterial fermentation of dietary fiber also change gut barrier integrity. ${ }^{38}$ Bacterial metabolites including SCFAs modulate brain and behavior via the vagus, and directly act on brain through circulation, which possibly causes comorbid psychiatric illness in IBS. $^{64}$

Peripheral CRF signaling is also activated by stress. The expression of CRF receptors and ligands are detected in various cells such as neuronal cells (enteric nervous system), enterochromaffin cells, and immune cells (mast cells, macrophages, dendritic cells, lymphocytes) in the colon. ${ }^{3,65,66}$ Although the mechanism of brain control of peripheral $\mathrm{CRF}$ secretion remains to be determined, the autonomic neural pathway is plausible. ${ }^{67}$ Activation of peripheral CRF receptors alters TJP via TLR4, causing colonic hyperpermeability. ${ }^{11,68}$ Impaired gut barrier induces bacterial translocation resulting in activation of local the immune system to induce dysbiosis and increase LPS. In turn, LPS activates TLR4 in immune cells including mast cells to trigger the production of proinflammatory cytokines, which induce visceral hypersensitivity, ${ }^{30}$ possibly through the activation of visceral afferent neurons. ${ }^{69}$ The proinflammatory cytokines also increase gut permeability via modifying $\mathrm{TJP}^{70}$ At the same time, CRF facilitates to release chemical mediators including proinflammatory cytokines from the mast cells, ${ }^{49}$ which can induce visceral hypersensitivity and impair gut barrier. ${ }^{10,50}$

LPS and proinflammatory cytokines can enter the circulation to act on the brain, which possibly may alter emotion and cognition. ${ }^{71,72}$ Moreover, proinflammatory cytokines act centrally in the brain to stimulate the secretion of CRF leading to activation of the HPA axis. ${ }^{73}$ Additionally, LPS not only stimulates TLR4 but also activates peripheral CRF receptors to cause colonic hyperpermeability. $^{30}$

Thus, CRF and TLR4-proinflammatory cytokine signaling create a vicious cycle to activate each other, resulting in impaired gut barrier and dysbiosis to cause the symptoms of IBS.

\section{Is There a Link Between Irritable Bowel Syndrome and Metabolic Syndrome?}

Recently, an importance of pathophysiological role of altered microbiota and impaired gut barrier has been recognized in MS similar to IBS

Impaired Gut Barrier and Dysbiosis: Role of Toll-like Receptor 4-Proinflammatory Cytokines in Metabolic Syndrome

It is well known that high-fat diet (HFD) induces MS. Although the mechanisms have not been precisely determined, HFD causes dysbiosis, leading to the impairment of the gut barrier. ${ }^{74,75}$ Butyrate derived from gut bacteria is involved in the maintenance of gut barrier, as described before, ${ }^{60}$ and HFD-induced dysbiosis reduces the production of butyrate in the gut. ${ }^{76}$ Additionally, oral supplementation of butyrate improves MS and dysbiosis induced by HFD in mice. ${ }^{77}$ In this context, HFD-induced reduction of butyrate may be one of the causes of leaky gut, which may be involved in MS.

Impaired gut barrier by HFD induces bacterial translocation, which can alter microbiota and activate the immune system to produce LPS. LPS, in turn reaches the systemic circulation, which is called metabolic endotoxemia, ${ }^{8}$ to cause low-grade inflammation and activate proinflammatory cytokine signaling via systemic TLR4 (Fig. 2). ${ }^{74,78}$ It was demonstrated that dysbiosis, increased intestinal inflammation, and decreased expression of TJP by HFD were 


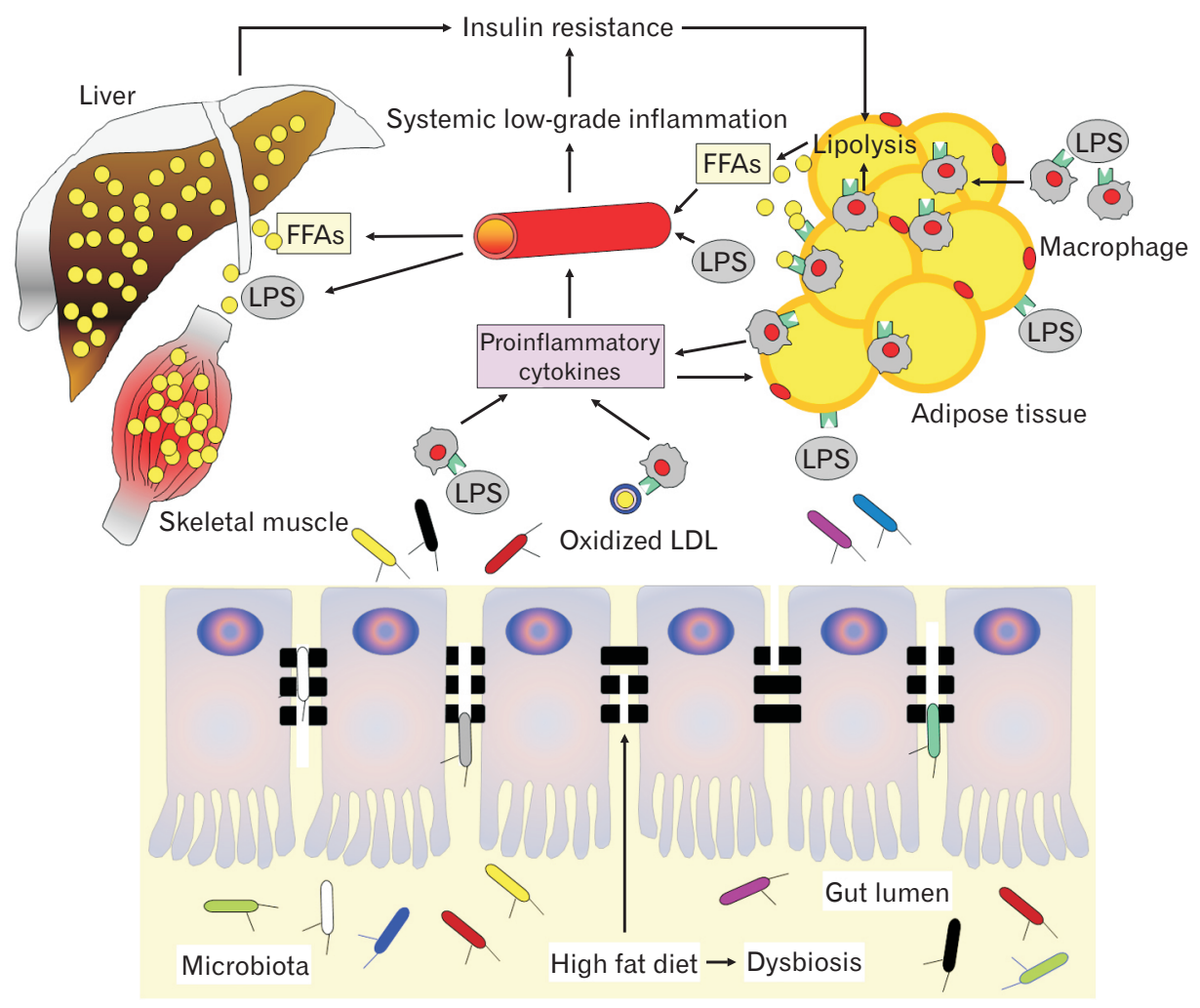

Figure 2. Schematic illustration of mechanisms of metabolic syndrome with special reference to Toll-like receptor 4 (TLR4) and proinflammatory cytokine signaling. High-fat diet impairs gut barrier to induce bacterial translocation resulting in dysbiosis. At the same time, dysbiosis reduces gut barrier integrity via the metabolites produced by gut bacteria. Additionally, this change activates the immune system to induce lipopolysaccharide (LPS). LPS activates systemic TLR4 to produce proinflammatory cytokines, resulting in low-grade inflammation, which causes insulin resistance. LPS triggers facilitatation to recruit macrophages into adipose tissue, and activates macrophages and adipocytes via TLR4 to induce local inflammation in adipose tissues. Under this condition, macrophages increase lipolysis through the release of proinflammatory cytokines to produce free fatty acids (FFAs). FFAs are delivered and accumulated in distant organs such as the liver and skeletal muscle, which can induce inflammation via TLR4, leading to insulin resistance. Among these FFAs, saturated FAs act as a ligand for TLR4 in both macrophages and adipocytes to increase the secretion of proinflammatory cytokines, which also contribute to insulin resistance. Additionally, insulin resistance at adipose tissue increases lipolysis, leading to increased release of FFAs. At the same time, oxidized low-density lipoprotein (LDL) is produced by oxidative stress induced by metabolic endotoxemia, and also activates TLR4 to produce proinflammatory cytokines, which is associated with insulin resistance. Thus, insulin resistance and inflammation cause a vicious cycle to induce each other via TLR4.

not observed in TLR4-deficient mice. ${ }^{79}$ These results suggest that dysbiosis and leaky gut induced by HFD may result from activated TLR4-proinflammatory cytokine signaling.

Obesity and insulin resistance are accompanied by low-grade inflammation, ${ }^{7,80}$ and TLR4 signaling is one of the main triggers of the obesity-induced inflammatory response. ${ }^{78}$ The elevated circulating levels of LPS and proinflammatory cytokines are observed in obese individuals. ${ }^{81,82}$ The expression of TLR4 is increased in muscle, visceral fat, and liver in obese and MS subjects. ${ }^{83}$ As macrophages having TLR4 infiltrate into these organs in obese subjects, increased expression of TLR4 is thought to be mainly due to macrophages. ${ }^{84,85}$
Infiltration of macrophages into adipose tissue is triggered by LPS, ${ }^{86}$ and macrophages interact with adipocytes to produce proinflammatory cytokines, ${ }^{87,88}$ thereby contributing to insulin resistance. $^{8}$ Additionally, TLR4 is highly expressed in adipocytes, and adipocytes per se produce proinflammatory cytokines triggered by LPS. $^{89}$

\section{Fatty Acids and Oxidized Low-density Lipoprotein Activate Toll-like Receptor 4 Signaling in Metabolic Syndrome}

Macrophages in adipose tissue can increase lipolysis through the release of proinflammatory cytokines, resulting in increased pro- 
duction of free fatty acids (FAAs). ${ }^{90}$ Then, the delivery of FFAs to the liver and skeletal muscle is increased, thereby causing the excessive accumulation of FFAs in these tissues. This change can induce inflammation via TLR4 signaling, leading to insulin resistance. ${ }^{91-94}$ At the same time, insulin resistance in adipose tissue increases lipolysis to facilitate the release of FFAs. Thus, insulin resistance and inflammation cause a vicious cycle to induce each other via TLR4 (Fig. 2).

Non-alcoholic fatty liver disease is defined as the ectopic accumulation of fat in the liver, not due to alcohol consumption, and is regarded as a hepatic manifestation of MS. Not only Kupffer cells, resident liver macrophages, but also stellate cells and hepatocytes express TLR4, and several studies demonstrated that TLR4 signaling plays a crucial role in this disease progression. ${ }^{95}$ At the same time, skeletal muscle cells have also TLR4 to produce proinflammatory cytokines in response to LPS. ${ }^{92,93}$

Among fatty acids (FAs) released from adipocytes triggered by macrophages, saturated FAs can act as a ligand for TLR4. ${ }^{96}$ Saturated FAs can activate TLR4 in both macrophages and adipocytes in adipose tissue to increase the secretion of proinflammatory cytokines, which possibly cause inflammation and are responsible for insulin resistance. ${ }^{78,97,98}$ Moreover, it was reported that dietary saturated FAs are associated with MS, and TLR4 signaling is necessary for saturated FAs to induce insulin resistance and obesity in animal models. ${ }^{99}$ Additionally, pharmacological inhibition of TLR4 inhibits fat-induced insulin resistance in rats. ${ }^{100}$

The association of MS and oxidative stress, a condition resulting from an imbalance between oxidant and antioxidant biological agents, has been known, ${ }^{101}$ and metabolic endotoxemia can elicit oxidative stress. ${ }^{102}$ Incidentally, the elevation of small and low-density lipoprotein (LDL) is common in MS, and these particles are easily oxidized by oxidative stress in MS, thereby producing oxidized LDL, which is atherogenic lipids. ${ }^{96}$ Oxidized LDL can activate TLR4 signaling such as saturated FAs, leading to the expression of proinflammatory cytokines, ${ }^{96}$ and high oxidized LDL levels are known to be associated with insulin resistance. ${ }^{103}$

These lines of evidence suggest that impaired gut barrier and dysbiosis with activation of TLR4-proinflammatory cytokine signaling by microbial LPS and non-microbial substances such as saturated FAs and oxidized LDL can cause insulin resistance to contribute to the pathophysiology of MS.

\section{Fatty Acids and Irritable Bowel Syndrome}

Polyunsaturated FAs also alter the activity of TLR4 signaling, and are involved in the risk of MS. Polyunsaturated FAs consist of
2 families ( $\omega-3$ and $\omega-6)$, and a high ratio of dietary $\omega-3 / \omega-6$ polyunsaturated FAs improves insulin resistance with decreased levels of circulating proinflammatory cytokines, and reduces TLR4 in skeletal muscle in both protein and gene levels in rats. ${ }^{104}$

Interestingly, there are several studies suggesting that FAs also play a role in the pathophysiology of IBS. Neonatal maternal separation induces visceral hypersensitivity and impairs gut barrier, ${ }^{105,106}$ and also increases plasma $\omega-6 / \omega-3$ polyunsaturated FAs ratio. ${ }^{107}$ Moreover, Chua et al, ${ }^{108}$ recently reported that higher proportions of plasma saturated FAs and lower proportion of total $\omega-3$ polyunsaturated FAs are associated with IBS.

\section{Corticotropin-releasing Factor and Metabolic Syndrome}

$\mathrm{CRF}$ and its related peptides are shown to be one of the key regulators of energy balance, and seem to contribute to the pathophysiology of MS. Genetic deletion of $\mathrm{CRF}_{2}$ increases energy expenditure with increased insulin sensitivity in HFD-induced obese mice. ${ }^{109} \mathrm{Ucn} 3$ is a selective ligand for $\mathrm{CRF}_{2}{ }^{110}$ and $\mathrm{Ucn}$ 3-null mice display improved insulin resistance increased by HFD diets. ${ }^{11}$ Moreover, overexpression of $\mathrm{U} \mathrm{cn} 3$ in the hypothalamus reduces insulin sensitivity. ${ }^{112}$ Additionally, stress-induced hypercortisolism, which is triggered by CRF, possibly mediates visceral obesity with insulin resistance and plays a pathophysiological role in MS. ${ }^{113,114}$

The evidence showing the role of CRF in the pathophysiology of MS has been scarce so far. However, as LPS activates CRF signaling, and CRF stimulates TLR4, as described before, ${ }^{11,30}$ metabolic endotoxemia, ie, high levels of LPS in blood observed in MS may activate CRF signaling, which may be involved in the pathophysiology of MS. Further studies are needed to explore this issue.

\section{Evidence for Pathophysiological Commonality Between Irritable Bowel Syndrome and Metabolic Syndrome}

In addition to the evidence above, there are several results which may also support the existence of pathophysiological commonality between IBS and MS. HFD-induced obesity increases expression of TLR4 in both brain and peripheral tissue with the increased level of proinflammatory cytokines, thereby causing visceral hypersensitivity in mice, ${ }^{115}$ suggesting the possibility that MS patients display visceral hypersensitivity like IBS via TLR4-proinflammatory cytokine signaling.

There have been several epidemiological studies investigating whether there is an association between obesity and IBS. Although 
the results are not fully consistent, the majority of these studies reported that prevalence of IBS was higher in obese subjects. ${ }^{116}$ Moreover, some studies also showed that weight loss intervention relieved the abdominal symptom severity in obese subjects with IBS. ${ }^{117}$ Lee, et al, ${ }^{118}$ demonstrated that increased visceral fat, but not subcutaneous fat was associated with an increased risk of IBS. As abdominal obesity, ie, increased visceral fat is one of the most prevalent manifestations of $\mathrm{MS},{ }^{119}$ this result may further support the notion that IBS and MS have a common mechanism. Conversely, there is also evidence that the frequency of MS is increased in IBS. ${ }^{120,121}$

On the basis of the evidence above, obesity or MS and IBS may be linked. However, it should be noted that these results do not always mean that activated TRL4-proinflammatory cytokine signaling is a common pathophysiological mechanism of MS and IBS. Several researchers showed that obese subjects had altered GI motility, ${ }^{122,123}$ which can cause the symptoms of IBS. Additionally, there is another possibility that a significant portion of IBS patients complains of food intolerance, ${ }^{124}$ which may affect the dietary pattern, thereby increasing frequency of MS. ${ }^{125}$ Further studies are needed to explore the mechanisms of link between IBS and MS.

\section{The Drugs Used to Treat Metabolic Syn- drome May Also Be Effective for Irritable Bowel Syndrome}

Based on this notion, we conjectured that the drugs used for MS and its components may also display favorable effects for IBS. Some of these drugs have pleiotropic effects contributing to reduce the risk of cardiovascular events, which are independent of primary action. It is well demonstrated that proinflammatory cytokines are associated with the risk of coronary heart disease independent of conventional risk factors. ${ }^{126}$ Thus, we attempted to determine the effects of several drugs used for MS having anti-cytokine properties, in visceral sensation and gut barrier in IBS models.

We found that liraglutide (a glucagon-like peptide-1 analog), pioglitazone, metformin, lovastatin (a $\beta$-Hydroxy $\beta$-methylglutarylCoA reductase inhibitor), and losartan (an angiotensin II type 1 receptor antagonist), which are prescribed for MS and its components, and all have anti-cytokine properties, blocked visceral hypersensitivity, and colonic hyperpermeability in IBS models, such as CRF, LPS, and repeated WAS. ${ }^{34-36,39,127}$ These results may further support the existence of link between IBS and MS via a common pathophysiological mechanism, ie, CRF-TLR4-proinflammatory cytokine signaling. Moreover, the results also suggest that these drugs for MS may improve the symptoms of IBS.

As the precise mechanisms of IBS have not been determined, only symptomatic treatments are the main in clinical practice. According to the evidence described above, inhibition of CRFTLR4-proinflammatory cytokine signaling is considered to be a novel approach for the treatment for IBS. However, biopharmaceutical agents to inhibit proinflammatory cytokine cannot be used because of their cost and side effects. Since these tested drugs above are widely prescribed, their application to IBS treatment seems not to be difficult. Large scale clinical trials to explore the effectiveness of these drugs in IBS treatment are needed in the future. Additionally, our results also suggest the possibility that these drugs may reduce the risk of cardiovascular events by improving the gut barrier via inhibiting proinflammatory cytokine signaling other than the primary mechanism.

\section{Conclusions}

IBS and MS may have common pathophysiology, ie, leaky gut and altered microbiota with activation of CRF-TLR4-proinflammatory cytokine signaling. This notion may pave the way for the development of novel treatment of IBS and MS, and help in a better understanding of the underlying mechanisms of these diseases.

\section{Financial support: None.}

\section{Conflicts of interest: None.}

Author contributions: Tsukasa Nozu reviewed the literature and drafted the original manuscript and figures; and Toshikatsu Okumura edited the manuscript and provided important intellectual directives.

\section{References}

1. Drossman DA, Camilleri M, Mayer EA, Whitehead WE. AGA technical review on irritable bowel syndrome. Gastroenterology 2002;123:2108-2131.

2. Taché Y, Kiank C, Stengel A. A role for corticotropin-releasing factor in functional gastrointestinal disorders. Curr Gastroenterol Rep 2009;11:270-277.

3. Nozu T, Okumura T. Corticotropin-releasing factor receptor type 1 and type 2 interaction in irritable bowel syndrome. J Gastroenterol 2015;50:819-830.

4. Taché Y, Million M. Role of corticotropin-releasing factor signaling in stress-related alterations of colonic motility and hyperalgesia. J Neurogastroenterol Motil 2015;21:8-24.

5. Lozano R, Naghavi M, Foreman K, et al. Global and regional mortal- 
ity from 235 causes of death for 20 age groups in 1990 and 2010: a systematic analysis for the Global Burden of Disease Study 2010. Lancet 2012;380:2095-2128

6. Festi D, Schiumerini R, Eusebi LH, Marasco G, Taddia M, Colecchia A. Gut microbiota and metabolic syndrome. World J Gastroenterol 2014;20:16079-16094.

7. Wellen KE, Hotamisligil GS. Inflammation, stress, and diabetes. J Clin Invest 2005;115:1111-1119.

8. Cani PD, Amar J, Iglesias MA, et al. Metabolic endotoxemia initiates obesity and insulin resistance. Diabetes 2007;56:1761-1772.

9. Mayer EA, Savidge T, Shulman RJ. Brain-gut microbiome interactions and functional bowel disorders. Gastroenterology 2014;146:1500-1512.

10. Camilleri M, Lasch K, Zhou W. Irritable bowel syndrome: methods, mechanisms, and pathophysiology. The confluence of increased permeability, inflammation, and pain in irritable bowel syndrome. Am J Physiol Gastrointest Liver Physiol 2012;303:G775-G785.

11. Nozu T, Miyagishi S, Nozu R, Takakusaki K, Okumura T. Altered colonic sensory and barrier functions by CRF: roles of TLR4 and IL-1. J Endocrinol 2018;239:241-252.

12. Cryan JF, O'Mahony SM. The microbiome-gut-brain axis: from bowel to behavior. Neurogastroenterol Motil 2011;23:187-192.

13. Fukudo S, Nomura T, Muranaka M, Taguchi F. Brain-gut response to stress and cholinergic stimulation in irritable bowel syndrome. A preliminary study. J Clin Gastroenterol 1993;17:133-141.

14. Mayer EA, Naliboff BD, Chang L, Coutinho SV. V. Stress and irritable bowel syndrome. Am J Physiol Gastrointest Liver Physiol 2001;280:G519-G524.

15. Fukudo S, Nomura T, Hongo M. Impact of corticotropin-releasing hormone on gastrointestinal motility and adrenocorticotropic hormone in normal controls and patients with irritable bowel syndrome. Gut 1998;42:845-849.

16. Lee C, Doo E, Choi JM, et al. The increased level of depression and anxiety in irritable bowel syndrome patients compared with healthy controls: systematic review and meta-analysis. J Neurogastroenterol Motil 2017;23:349-362.

17. Fond G, Loundou A, Hamdani N, et al. Anxiety and depression comorbidities in irritable bowel syndrome (IBS): a systematic review and meta-analysis. Eur Arch Psychiatry Clin Neurosci 2014;264:651-660.

18. Kellow JE, Delvaux M, Azpiroz F, Camilleri M, Quigley EM, Thompson DG. Principles of applied neurogastroenterology: physiology/motility-sensation. Gut 1999;45(suppl 2):II17-II24 .

19. Delvaux M. Role of visceral sensitivity in the pathophysiology of irritable bowel syndrome. Gut 2002;51(suppl 1):i67-i71.

20. Mertz H, Naliboff B, Munakata J, Niazi N, Mayer EA. Altered rectal perception is a biological marker of patients with irritable bowel syndrome. Gastroenterology 1995;109:40-52.

21. Bouin M, Plourde V, Boivin M, et al. Rectal distention testing in patients with irritable bowel syndrome: sensitivity, specificity, and predictive values of pain sensory thresholds. Gastroenterology 2002;122:17711777.

22. Kanazawa M, Hongo M, Fukudo S. Visceral hypersensitivity in irritable bowel syndrome. J Gastroenterol Hepatol 2011;26(suppl 3):119-121.
23. Nozu T, Kudaira M, Kitamori S, Uehara A. Repetitive rectal painful distention induces rectal hypersensitivity in patients with irritable bowel syndrome. J Gastroenterol 2006;41:217-222.

24. Farzaei MH, Bahramsoltani R, Abdollahi M, Rahimi R. The role of visceral hypersensitivity in irritable bowel syndrome: pharmacological targets and novel treatments. J Neurogastroenterol Motil 2016;22:558574.

25. Camilleri M, Gorman H. Intestinal permeability and irritable bowel syndrome. Neurogastroenterol Motil 2007;19:545-552.

26. Dlugosz A, Nowak P, D'Amato M, et al. Increased serum levels of lipopolysaccharide and antiflagellin antibodies in patients with diarrheapredominant irritable bowel syndrome. Neurogastroenterol Motil 2015;27:1747-1754.

27. Ortiz-Lucas M, Saz-Peiró P, Sebastián-Domingo JJ. Irritable bowel syndrome immune hypothesis. Part two: the role of cytokines. Rev Esp Enferm Dig 2010;102:711-717.

28. Sinagra E, Pompei G, Tomasello G, et al. Inflammation in irritable bowel syndrome: myth or new treatment target? World J Gastroenterol 2016;22:2242-2255.

29. Liebregts T, Adam B, Bredack C, et al. Immune activation in patients with irritable bowel syndrome. Gastroenterology 2007;132:913-920.

30. Nozu T, Miyagishi S, Nozu R, Takakusaki K, Okumura T. Lipopolysaccharide induces visceral hypersensitivity: role of interleukin-1, interleukin-6, and peripheral corticotropin-releasing factor in rats. J Gastroenterol 2017;52:72-80.

31. Avila M, Gonzalez-Espinosa C. Signaling through toll-like receptor 4 and mast cell-dependent innate immunity responses. IUBMB Life 2011;63:873-880.

32. Boyer J, Saint-Paul MC, Dadone B, et al. Inflammatory cell distribution in colon mucosa as a new tool for diagnosis of irritable bowel syndrome: a promising pilot study. Neurogastroenterol Motil 2018;30.

33. Creekmore AL, Hong S, Zhu S, Xue J, Wiley JW. Chronic stressassociated visceral hyperalgesia correlates with severity of intestinal barrier dysfunction. Pain 2018;159:1777-1789.

34. Nozu T, Miyagishi S, Kumei S, Nozu R, Takakusaki K, Okumura T. Lovastatin inhibits visceral allodynia and increased colonic permeability induced by lipopolysaccharide or repeated water avoidance stress in rats. Eur J Pharmacol 2017;818:228-234.

35. Nozu T, Miyagishi S, Nozu R, Takakusaki K, Okumura T. Pioglitazone improves visceral sensation and colonic permeability in a rat model of irritable bowel syndrome. J Pharmacol Sci 2018;139:46-49.

36. Nozu T, Miyagishi S, Kumei S, Nozu R, Takakusaki K, Okumura T. Metformin inhibits visceral allodynia and increased gut permeability induced by stress in rats. J Gastroenterol Hepatol 2019;34:186-193.

37. Nozu T, Miyagishi S, Nozu R, Takakusaki K, Okumura T. Dehydroepiandrosterone sulfate improves visceral sensation and gut barrier in a rat model of irritable bowel syndrome. Eur J Pharmacol 2019;852:198206.

38. Nozu T, Miyagishi S, Nozu R, Takakusaki K, Okumura T. Butyrate inhibits visceral allodynia and colonic hyperpermeability in rat models of irritable bowel syndrome. Sci Rep 2019;9:19603.

39. Nozu T, Miyagishi S, Nozu R, Takakusaki K, Okumura T. Losartan 
improves visceral sensation and gut barrier in a rat model of irritable bowel syndrome. Neurogastroenterol Motil 2020;32:e13819.

40. Nozu T, Kudaira M. Corticotropin-releasing factor induces rectal hypersensitivity after repetitive painful rectal distention in healthy humans. J Gastroenterol 2006;41:740-744.

41. Barreau F, Cartier C, Leveque M, et al. Pathways involved in gut mucosal barrier dysfunction induced in adult rats by maternal deprivation: corticotrophin-releasing factor and nerve growth factor interplay. J Physiol 2007;580(pt 1):347-356.

42. Santos J, Saunders PR, Hanssen NP, et al. Corticotropin-releasing hormone mimics stress-induced colonic epithelial pathophysiology in the rat. Am J Physiol 1999;277:G391-G399.

43. Saunders PR, Santos J, Hanssen NP, Yates D, Groot JA, Perdue MH. Physical and psychological stress in rats enhances colonic epithelial permeability via peripheral CRH. Dig Dis Sci 2002;47:208-215.

44. Söderholm JD, Yates DA, Gareau MG, Yang PC, MacQueen G, Perdue MH. Neonatal maternal separation predisposes adult rats to colonic barrier dysfunction in response to mild stress. Am J Physiol Gastrointest Liver Physiol 2002;283:G1257-G1263.

45. Hillhouse EW, Grammatopoulos DK. The molecular mechanisms underlying the regulation of the biological activity of corticotropin-releasing hormone receptors: implications for physiology and pathophysiology. Endocr Rev 2006;27:260-286.

46. Perrin MH, Vale WW. Corticotropin releasing factor receptors and their ligand family. Ann N Y Acad Sci 1999;885:312-328.

47. Nozu T, Takakusaki K, Okumura T. A balance theory of peripheral corticotropin-releasing factor receptor type 1 and type 2 signaling to induce colonic contractions and visceral hyperalgesia in rats. Endocrinology 2014;155:4655-4664.

48. Nozu T, Miyagishi S, Nozu R, Takakusaki K, Okumura T. Repeated water avoidance stress induces visceral hypersensitivity: role of IL1, IL-6 and peripheral corticotropin-releasing factor. J Gastroenterol Hepatol 2017;32:1958-1965.

49. Overman EL, Rivier JE, Moeser AJ. CRF induces intestinal epithelial barrier injury via the release of mast cell proteases and TNF-alpha. PLoS One 2012;7:e39935.

50. Barbara G, Wang B, Stanghellini V, et al. Mast cell-dependent excitation of visceral-nociceptive sensory neurons in irritable bowel syndrome. Gastroenterology 2007;132:26-37.

51. Bhattarai Y, Muniz Pedrogo DA, Kashyap PC. Irritable bowel syndrome: a gut microbiota-related disorder? Am J Physiol Gastrointest Liver Physiol 2017;312:G52-G62.

52. Tap J, Derrien M, Törnblom H, et al. Identification of an intestinal microbiota signature associated with severity of irritable bowel syndrome. Gastroenterology 2017;152:111-123, e8.

53. O'Mahony SM, Marchesi JR, Scully P, et al. Early life stress alters behavior, immunity, and microbiota in rats: implications for irritable bowel syndrome and psychiatric illnesses. Biol Psychiatry 2009;65:263-267.

54. Xu D, Gao J, Gillilland M 3rd, et al. Rifaximin alters intestinal bacteria and prevents stress-induced gut inflammation and visceral hyperalgesia in rats. Gastroenterology 2014;146:484-496, e4.

55. Salguero MV, Al-Obaide MAI, Singh R, Siepmann T, Vasylyeva TL.
Dysbiosis of gram-negative gut microbiota and the associated serum lipopolysaccharide exacerbates inflammation in type 2 diabetic patients with chronic kidney disease. Exp Ther Med 2019;18:3461-3469.

56. Bailey MT, Dowd SE, Galley JD, Hufnagle AR, Allen RG, Lyte M. Exposure to a social stressor alters the structure of the intestinal microbiota: implications for stressor-induced immunomodulation. Brain Behav Immun 2011;25:397-407.

57. Santos J, Yang PC, Söderholm JD, Benjamin M, Perdue MH. Role of mast cells in chronic stress induced colonic epithelial barrier dysfunction in the rat. Gut 2001;48:630-636.

58. Ait-Belgnaoui A, Durand H, Cartier C, et al. Prevention of gut leakiness by a probiotic treatment leads to attenuated HPA response to an acute psychological stress in rats. Psychoneuroendocrinology 2012;37:18851895.

59. Kelly JR, Kennedy PJ, Cryan JF, Dinan TG, Clarke G, Hyland NP. Breaking down the barriers: the gut microbiome, intestinal permeability and stress-related psychiatric disorders. Front Cell Neurosci 2015;9:392.

60. Plöger S, Stumpff F, Penner GB, et al. Microbial butyrate and its role for barrier function in the gastrointestinal tract. Ann N Y Acad Sci 2012;1258:52-59.

61. Zhang J, Song L, Wang Y, et al. Beneficial effect of butyrate-producing Lachnospiraceae on stress-induced visceral hypersensitivity in rats. J Gastroenterol Hepatol 2019;34:1368-1376.

62. Crouzet L, Gaultier E, Del'Homme C, et al. The hypersensitivity to colonic distension of IBS patients can be transferred to rats through their fecal microbiota. Neurogastroenterol Motil 2013;25:e272-e282.

63. Zong Y, Zhu S, Zhang S, Zheng G, Wiley JW, Hong S. Chronic stress and intestinal permeability: lubiprostone regulates glucocorticoid receptor-mediated changes in colon epithelial tight junction proteins, barrier function, and visceral pain in the rodent and human. Neurogastroenterol Motil 2019:e13477.

64. Cryan JF, Dinan TG. Mind-altering microorganisms: the impact of the gut microbiota on brain and behaviour. Nat Rev Neurosci 2012;13:701712.

65. Audhya T, Jain R, Hollander CS. Receptor-mediated immunomodulation by corticotropin-releasing factor. Cell Immunol 1991;134:77-84.

66. Hojo M, Ohkusa T, Tomeoku H, et al. Corticotropin-releasing factor secretion from dendritic cells stimulated by commensal bacteria. World J Gastroenterol 2011;17:4017-4022.

67. Zheng PY, Feng BS, Oluwole C, et al. Psychological stress induces eosinophils to produce corticotrophin releasing hormone in the intestine. Gut 2009;58:1473-1479.

68. Yu Y, Liu ZQ, Liu XY, et al. Stress-derived corticotropin releasing factor breaches epithelial endotoxin tolerance. PLoS One 2013;8:e65760.

69. Obreja O, Rathee PK, Lips KS, Distler C, Kress M. IL-1 beta potentiates heat-activated currents in rat sensory neurons: involvement of IL1RI, tyrosine kinase, and protein kinase C. FASEB J 2002;16:14971503.

70. Suzuki T, Yoshinaga N, Tanabe S. Interleukin-6 (IL-6) regulates claudin-2 expression and tight junction permeability in intestinal epithelium. J Biol Chem 2011;286:31263-31271.

71. Liu L, Zhu G. Gut-brain axis and mood disorder. Front Psychiatry 
2018;9:223.

72. Calarge CA, Devaraj S, Shulman RJ. Gut permeability and depressive symptom severity in unmedicated adolescents. J Affect Disord 2019;246:586-594.

73. Uehara A, Gottschall PE, Dahl RR, Arimura A. Interleukin-1 stimulates $\mathrm{ACTH}$ release by an indirect action which requires endogenous corticotropin releasing factor. Endocrinology 1987;121:1580-1582.

74. Dabke K, Hendrick G, Devkota S. The gut microbiome and metabolic syndrome. J Clin Invest 2019;129:4050-4057.

75. Araújo JR, Tomas J, Brenner C, Sansonetti PJ. Impact of high-fat diet on the intestinal microbiota and small intestinal physiology before and after the onset of obesity. Biochimie 2017;141:97-106.

76. Jakobsdottir G, Xu J, Molin G, Ahrné S, Nyman M. High-fat diet reduces the formation of butyrate, but increases succinate, inflammation, liver fat and cholesterol in rats, while dietary fibre counteracts these effects. PLoS One 2013;8:e80476.

77. Gao F, Lv YW, Long J, et al. Butyrate improves the metabolic disorder and gut microbiome dysbiosis in mice induced by a high-fat diet. Front Pharmacol 2019;10:1040.

78. Rogero MM, Calder PC. Obesity, inflammation, toll-like receptor 4 and fatty acids. Nutrients 2018;10:432.

79. Kim KA, Gu W, Lee IA, Joh EH, Kim DH. High fat diet-induced gut microbiota exacerbates inflammation and obesity in mice via the TLR4 signaling pathway. PLoS One 2012;7:e47713.

80. Rehman K, Akash MS. Mechanisms of inflammatory responses and development of insulin resistance: how are they interlinked? J Biomed Sci 2016;23:87.

81. Gregor MF, Hotamisligil GS. Inflammatory mechanisms in obesity. Annu Rev Immunol 2011;29:415-445.

82. Kallio KA, Hätönen KA, Lehto M, Salomaa V, Männistö S, Pussinen PJ. Endotoxemia, nutrition, and cardiometabolic disorders. Acta Diabetol 2015;52:395-404.

83. Jialal I, Kaur H, Devaraj S. Toll-like receptor status in obesity and metabolic syndrome: a translational perspective. J Clin Endocrinol Metab 2014;99:39-48.

84. Miura K, Ishioka M, Iijima K. The roles of the gut microbiota and toll-like receptors in obesity and nonalcoholic fatty liver disease. J Obes Metab Syndr 2017;26:86-96.

85. Ahmad R, Al-Mass A, Atizado V, et al. Elevated expression of the toll like receptors 2 and 4 in obese individuals: its significance for obesityinduced inflammation. J Inflamm (Lond) 2012;9:48.

86. Nakarai H, Yamashita A, Nagayasu S, et al. Adipocyte-macrophage interaction may mediate LPS-induced low-grade inflammation: potential link with metabolic complications. Innate Immun 2012;18:164-170.

87. Hotamisligil GS, Shargill NS, Spiegelman BM. Adipose expression of tumor necrosis factor-alpha: direct role in obesity-linked insulin resistance. Science 1993;259:87-91.

88. Weisberg SP, McCann D, Desai M, Rosenbaum M, Leibel RL, Ferrante AW Jr. Obesity is associated with macrophage accumulation in adipose tissue. J Clin Invest 2003;112:1796-1808.

89. Lin Y, Lee H, Berg AH, Lisanti MP, Shapiro L, Scherer PE. The lipopolysaccharide-activated toll-like receptor (TLR)-4 induces syn- thesis of the closely related receptor TLR-2 in adipocytes. J Biol Chem 2000;275:24255-24263.

90. Daemen S, Schilling JD. The interplay between tissue niche and macrophage cellular metabolism in obesity. Front Immunol 2019;10:3133.

91. Farrell GC, van Rooyen D, Gan L, Chitturi S. NASH is an inflammatory disorder: pathogenic, prognostic and therapeutic implications. Gut Liver 2012;6:149-171.

92. Frost RA, Nystrom GJ, Lang CH. Lipopolysaccharide stimulates nitric oxide synthase-2 expression in murine skeletal muscle and $\mathrm{C}_{2} \mathrm{C}_{12}$ myoblasts via toll-like receptor-4 and c-Jun $\mathrm{NH}(2)$-terminal kinase pathways. Am J Physiol Cell Physiol 2004;287:C1605-C1615.

93. Frost RA, Nystrom GJ, Lang CH. Lipopolysaccharide and proinflammatory cytokines stimulate interleukin-6 expression in C2C12 myoblasts: role of the Jun $\mathrm{NH}_{2}$-terminal kinase. Am J Physiol Regul Integr Comp Physiol 2003;285:R1153-R1164.

94. Williams KH, Shackel NA, Gorrell MD, McLennan SV, Twigg SM. Diabetes and nonalcoholic fatty liver disease: a pathogenic duo. Endocr Rev 2013;34:84-129.

95. Kolodziejczyk AA, Zheng D, Shibolet O, Elinav E. The role of the microbiome in NAFLD and NASH. EMBO Mol Med 2019;11:e9302.

96. Rocha DM, Caldas AP, Oliveira LL, Bressan J, Hermsdorff HH. Saturated fatty acids trigger TLR4-mediated inflammatory response. Atherosclerosis 2016;244:211-215.

97. Suganami T, Tanimoto-Koyama K, Nishida J, et al. Role of the toll-like receptor 4/NF-kappaB pathway in saturated fatty acid-induced inflammatory changes in the interaction between adipocytes and macrophages. Arterioscler Thromb Vasc Biol 2007;27:84-91.

98. Shi H, Kokoeva MV, Inouye K, Tzameli I, Yin H, Flier JS. TLR4 links innate immunity and fatty acid-induced insulin resistance. J Clin Invest 2006;116:3015-3025.

99. Fessler MB, Rudel LL, Brown JM. Toll-like receptor signaling links dietary fatty acids to the metabolic syndrome. Curr Opin Lipidol 2009;20:379-385.

100. Zhang N, Liang H, Farese RV, Li J, Musi N, Hussey SE. Pharmacological TLR4 inhibition protects against acute and chronic fat-induced insulin resistance in rats. PLoS One 2015;10:e0132575.

101. Hopps E, Noto D, Caimi G, Averna MR. A novel component of the metabolic syndrome: the oxidative stress. Nutr Metab Cardiovasc Dis 2010;20:72-77.

102. Neves AL, Coelho J, Couto L, Leite-Moreira A, Roncon-Albuquerque $\mathrm{R}$ Jr. Metabolic endotoxemia: a molecular link between obesity and cardiovascular risk. J Mol Endocrinol 2013;51:R51-R64.

103. Linna MS, Ahotupa M, Kukkonen-Harjula K, Fogelholm M, Vasankari TJ. Co-existence of insulin resistance and high concentrations of circulating oxidized LDL lipids. Ann Med 2015;47:394-398.

104. Liu HQ, Qiu Y, Mu Y, et al. A high ratio of dietary n-3/n-6 polyunsaturated fatty acids improves obesity-linked inflammation and insulin resistance through suppressing activation of TLR4 in SD rats. Nutr Res 2013;33:849-858.

105. Barreau F, Ferrier L, Fioramonti J, Bueno L. Neonatal maternal deprivation triggers long term alterations in colonic epithelial barrier and mucosal immunity in rats. Gut 2004;53:501-506. 
106. Yi L, Zhang H, Sun H, et al. Maternal separation induced visceral hypersensitivity from childhood to adulthood. J Neurogastroenterol Motil 2017;23:306-315.

107. Clarke G, O'Mahony SM, Hennessy AA, et al. Chain reactions: earlylife stress alters the metabolic profile of plasma polyunsaturated fatty acids in adulthood. Behav Brain Res 2009;205:319-321.

108. Chua CS, Huang SY, Cheng CW, et al. Fatty acid components in Asian female patients with irritable bowel syndrome. Medicine (Baltimore) 2017;96:e9094.

109. Bale TL, Anderson KR, Roberts AJ, Lee KF, Nagy TR, Vale WW. Corticotropin-releasing factor receptor-2-deficient mice display abnormal homeostatic responses to challenges of increased dietary fat and cold. Endocrinology 2003;144:2580-2587.

110. Taché Y, Bonaz B. Corticotropin-releasing factor receptors and stressrelated alterations of gut motor function. J Clin Invest 2007;117:33-40.

111. Li C, Chen P, Vaughan J, Lee KF, Vale W. Urocortin 3 regulates glucose-stimulated insulin secretion and energy homeostasis. Proc Natl Acad Sci USA 2007;104:4206-4211.

112. Kuperman Y, Issler O, Regev L, et al. Perifornical urocortin-3 mediates the link between stress-induced anxiety and energy homeostasis. Proc Natl Acad Sci USA 2010;107:8393-8398.

113. Chrousos GP. The role of stress and the hypothalamic-pituitary-adrenal axis in the pathogenesis of the metabolic syndrome: neuro-endocrine and target tissue-related causes. Int J Obes Relat Metab Disord 2000;24(suppl 2):S50-S55.

114. Rosmond R. Role of stress in the pathogenesis of the metabolic syndrome. Psychoneuroendocrinology 2005;30:1-10.

115. Tramullas M, Finger BC, Dinan TG, Cryan JF. Obesity takes its toll on visceral pain: high-fat diet induces toll-like receptor 4-dependent visceral hypersensitivity. PLoS One 2016;11:e0155367.

116. Pickett-Blakely O. Obesity and irritable bowel syndrome: a comprehensive review. Gastroenterol Hepatol (NY) 2014;10:411-416.
117. Aasbrenn M, Lydersen S, Farup PG. A conservative weight loss intervention relieves bowel symptoms in morbidly obese subjects with irritable bowel syndrome: a prospective cohort study. J Obes 2018;2018:3732753.

118. Lee CG, Lee JK, Kang YS, et al. Visceral abdominal obesity is associated with an increased risk of irritable bowel syndrome. Am J Gastroenterol 2015;110:310-319.

119. Després JP, Lemieux I. Abdominal obesity and metabolic syndrome. Nature 2006;444:881-887.

120. Guo Y, Niu K, Momma H, et al. Irritable bowel syndrome is positively related to metabolic syndrome: a population-based cross-sectional study. PLoS One 2014;9:e112289.

121. Cholongitas E, Pipili C, Dasenaki M. Gastro-oesophageal reflux disease and irritable bowel syndrome significantly associated with metabolic syndrome. Scand J Gastroenterol 2008;43:1405-1406.

122. Basilisco G, Camboni G, Bozzani A, Vita P, Doldi S, Bianchi PA. Orocecal transit delay in obese patients. Dig Dis Sci 1989;34:509-512.

123. Sadik R, Björnsson E, Simrén M. The relationship between symptoms, body mass index, gastrointestinal transit and stool frequency in patients with irritable bowel syndrome. Eur J Gastroenterol Hepatol 2010;22:102-108.

124. Eswaran S, Tack J, Chey WD. Food: the forgotten factor in the irritable bowel syndrome. Gastroenterol Clin North Am 2011;40:141-162.

125. Chirila I, Petrariu FD, Ciortescu I, Mihai C, Drug VL. Diet and irritable bowel syndrome. J Gastrointestin Liver Dis 2012;21:357-362.

126. Kaptoge S, Seshasai SR, Gao P, et al. Inflammatory cytokines and risk of coronary heart disease: new prospective study and updated metaanalysis. Eur Heart J 2014;35:578-589.

127. Nozu T, Miyagishi S, Kumei S, Nozu R, Takakusaki K, Okumura T. Glucagon-like peptide-1 analog, liraglutide improves visceral sensation and gut permeability in rats. J Gastroenterol Hepatol 2018;33:232-239. 